\title{
Vaccine-induced monoclonal antibodies targeting circumsporozoite protein prevent Plasmodium falciparum infection
}

\author{
Lander Foquet, ${ }^{1}$ Cornelus C. Hermsen,,2 Geert-Jan van Gemert, ${ }^{2}$ Eva Van Braeckel,1 \\ Karin E. Weening, ${ }^{1}$ Robert Sauerwein,, ${ }^{2}$ Philip Meuleman, ${ }^{1}$ and Geert Leroux-Roels ${ }^{1}$ \\ ${ }^{1}$ Center for Vaccinology, Ghent University, Ghent, Belgium. ${ }^{2}$ Radboud University Nijmegen, Medical Centre, Nijmegen, The Netherlands.
}

\begin{abstract}
Malaria, which is the result of Plasmodium falciparum infection, is a global health threat that resulted in 655,000 deaths and 216 million clinical cases in 2010 alone. Recent phase 3 trials with malaria vaccine candidate RTS,S/AS01 (RTS,S) in children has demonstrated modest efficacy against clinical and severe malaria. RTS,S targets the pre-erythrocytic phase of the disease and induces high antibody titers against the $P$. falciparum circumsporozoite protein (CSP) and a moderate $\mathrm{CD4}^{+} \mathrm{T}$ cell response. The individual contribution of these adaptive immune responses to protection from infection remains unknown. Here, we found that prophylactic administration of anti-CSP $\mathrm{mAbs}$ derived from an RTS,S-vaccinated recipient fully protected mice with humanized livers from i.v.- and mosquito bite-delivered $P$. falciparum sporozoite challenge. Titers of anti-CSP that conveyed full protection were within the range observed in human RTS,S vaccine recipients. Increasing anti-CSP titers resulted in a dose-dependent reduction of the liver parasite burden. These data indicate that RTS,S-induced antibodies are protective and provide sterilizing immunity against $P$. falciparum infection when reaching or exceeding a critical plasma concentration.
\end{abstract}

\section{Introduction}

Plasmodium species have developed multiple strategies to evade and suppress host immunity, which makes treatment and vaccine development very difficult (1). During a blood meal, an infected mosquito injects around 100 sporozoites into the skin, from which the parasites migrate to the bloodstream and travel to the liver $(2,3)$. After invasion of a hepatocyte, the parasite enters the pre-erythrocytic stage, which lasts 6.5 days (4). The study of Plasmodium falciparum's liver stage is hampered by the low in vitro infection rate of human or primate host cells and by the need for a specialized insectary to rear and infect Anopheles mosquitoes for the production of sporozoites. The development of a mouse model with fully functional human hepatocytes has made it possible to study the liver stage in a preclinical in vivo setting (5-11).

Several candidate malaria vaccines are in development, but most study results have been rather disappointing (12-15). In phase 3 clinical trials, the most advanced malaria vaccine candidate, RTS,S/AS01 (GSK Vaccines; referred to herein as RTS,S), has shown $31 \%$ and $50 \%$ protective efficacy against clinical malaria in infants $(6-12$ weeks old) and children (5-17 months old), respectively $(16,17)$. RTS, $S$ is based on the hepatitis B surface antigen (HBsAg) and the P. falciparum circumsporozoite protein (CSP) antigen virus-like particle (VLP) platform (1). In vaccinated humans, RTS,S induces high IgG concentrations to the NANP $\mathrm{CSP}$ repeat region and $\mathrm{CD} 4^{+} \mathrm{T}$ cells that interfere with the ability of the malaria sporozoites to infect hepatocytes (pre-erythrocytic stage) $(1,18)$. The exact mechanism of protection is still unknown, and in vitro correlates of protection have not yet been defined. Although the titer of anti-CSP IgG is not an established correlate of protection, an association with efficacy has been observed in

Conflict of interest: The authors have declared that no conflict of interest exists. Citation for this article: J Clin Invest. 2014;124(1):140-144. doi:10.1172/JCI70349. several trials $(15,19-23)$, and it is suggested that the protective threshold for anti-CSP IgG concentrations in plasma is $>20 \mu \mathrm{g} / \mathrm{ml}$ (24). In addition, an independent and weaker association between CSP-specific $\mathrm{CD}^{+} \mathrm{T}$ cell responses and protection was observed in 2 phase II trials of RTS,S vaccines $(21,23)$.

\section{Results and Discussion}

To evaluate the protective efficacy of anti-CSP IgG in the absence of any confounding (i.e., T cell-mediated) factors, we administered varying doses of 3 human anti-CSP mAbs (designated Mal1C, Mal2A, and Mal3B) into humanized uPA-SCID mice before exposure to $P$. falciparum. The human mAbs recognizing the NANP repeat region of CSP were derived from a subject vaccinated with RTS,S, as described previously (25). uPA-SCID mice were transplanted with cryopreserved primary human hepatocytes, all from the same lot, as described previously (5). The capacity of antiCSP mAbs to interact with sporozoites and inhibit their motility and cell traversal has previously been examined in vitro using a gliding assay (26) and an inhibition of sporozoite invasion assay $(27,28)$. The RTS,S-induced anti-CSP mAb Mal1C dose-dependently inhibited gliding motility, with complete inhibition at $1,000 \mathrm{EU} / \mathrm{ml}$ (Figure 1, A, C, and D). In the inhibition of sporozoite invasion assay, parasites were added to wells containing HepG2 or Huh7.5 cells. The frequency of parasites invading Huh7.5 and HepG2 cells (27) was comparable (Figure 1, B, E, and F). Since both assays allow for a long interaction time between antibody and sporozoite, one may wonder whether antibody concentrations that inhibit sporozoite functions in vitro are also effective in vivo, when antibodies and sporozoites can only interact during the brief passage from the mosquito bite to the liver (29).

To examine whether human anti-CSP mAbs are capable of preventing in vivo infection with $P$. falciparum sporozoites, 13 humanized uPA-SCID mice were injected i.p. with PBS, and 

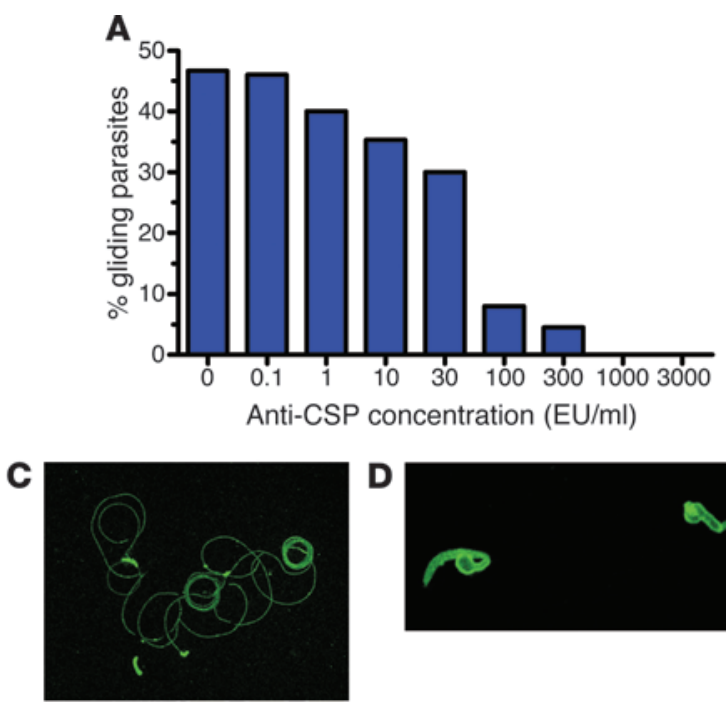

\section{B}

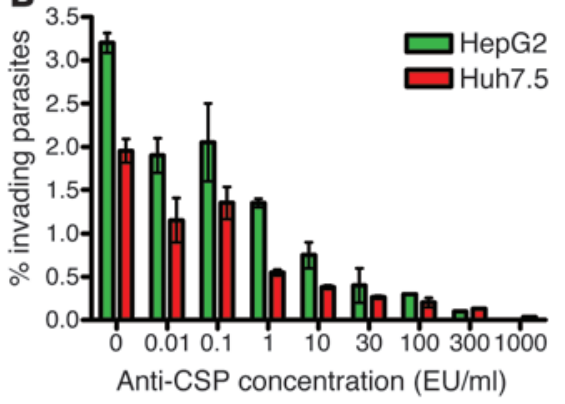

E
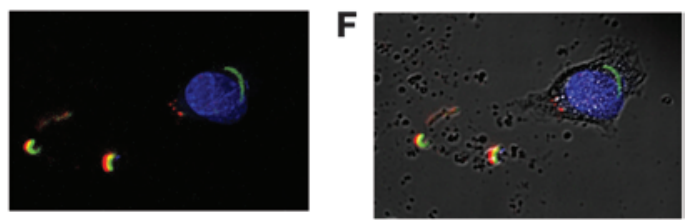

\section{Figure 1}

In vitro analysis of functional effects of RTS,S vaccine-induced mAbs on P. falciparum sporozoites. (A and B) A gliding motility assay (A) and an inhibition of sporozoite invasion assay (B; HepG2 and Huh7.5 cells) were performed after preincubation with different concentrations of Mal1C. Data represent mean \pm SD from triplicate wells. (C and D) Gliding assay. Shown are images of the trails produced by sporozoites in the absence (C) and presence (D) of anti-CSP mAbs (1,000 EU/ml). (E and F) Inhibition of sporozoite invasion assay. (E) Merged image showing extracellular (red and green) and intracellular (green) parasites. Nuclei were stained with DAPI (blue). (F) Additional overlay with a light microscopic image shows the cell membrane. Original magnification, $\times 63$.

17 mice were given varying doses of the anti-CSP mAb Mal1C: $2 \mathrm{mg}(n=11), 200 \mu \mathrm{g}(n=3)$, and $20 \mu \mathrm{g}(n=3)$. The following day, plasma concentrations of circulating mAbs were measured using a validated and standardized ELISA (25). Immediately thereafter, mice were challenged either via i.v. injection of 150,000 sporozoites (7 PBS, 6 Mal1C) or by exposing each mouse to 20 P. falciparum-infected mosquitoes that were allowed to feed for 20 minutes (6 PBS, 11 Mal1C) (12). 5 days after challenge, mice were euthanized, and their livers were divided into 12 standardized sections. From each of these fragments, $25 \mathrm{mg}$ was used to determine the human hepatocyte content and the P. falciparum liver load using quantitative RT-PCR (qPCR; L. Foquet, unpublished observations, and refs. 30, 31). Regardless of infection route, all PBS-treated mice were infected with $P$. falciparum, and all mice pretreated with $2 \mathrm{mg}$ Mal1C were protected (Table 1). AntiCSP plasma concentrations (geometric mean titer [GMT]) measured immediately before challenge were 3,421.5 and 3,133.4 EU/ $\mathrm{ml}$ in the i.v.- and mosquito bite-infected groups, respectively. After injection of $200 \mu \mathrm{g}$ Mal1C, 2 of 3 mice were protected from infection. Antibody concentrations measured in the protected mice before infection were 139.7 and $273.1 \mathrm{EU} / \mathrm{ml}$. The single unprotected mouse had an anti-CSP titer of $230.0 \mathrm{EU} / \mathrm{ml}$ and showed a much lower liver parasite burden than sham-treated mice (29.4 P. falciparum $/ 10^{6}$ human hepatocytes; Table 1). Liver parasite burden after treatment with $20 \mu \mathrm{g}$ mAb in 3 mice showed minimal reduction compared with sham-treated animals. Anti-CSP mAb concentrations in these mice were 31.5, 23.7, and 13.1 EU/ml (Table 1). Next, we tested whether 2 additional mAbs are able to prevent $P$. falciparum infection when administered in a dose corresponding to serum concentrations achievable by RTS,S vaccination $(18,32)$. Both Mal2A (HV3-HD3-HJ4:KV3-KJ2) and Mal3B (HV3-HD1-HJ6:KV1-KJ1) were different from Mal1C
(HV3-HD3-HJ4:KV2-KJ2), as determined by sequence analysis of $V_{H}: V_{L}$ pairs (33). Groups of 3 mice were injected i.p. with $400 \mu \mathrm{g}$ of Mal1C, Mal2A, or Mal3B and challenged the next day by infected mosquito bites (Table 1). The anti-CSP plasma concentrations (GMT) measured before infection were 668.1, 723.1, and $868.4 \mathrm{EU} / \mathrm{ml}$, respectively. As a control, 1 humanized mouse was injected with PBS and 1 with $400 \mu \mathrm{g}$ of a control mAb directed against HBsAg, as anti-HBsAg antibodies are also induced by RTS,S. All mice treated with the anti-CSP mAbs were protected against infection, whereas both control mice were infected at day 5 after challenge.

Previous research showed that passive transfer of antibodies directed against the repeat region of $P$. berghei CSP is capable of arresting $P$. berghei sporozoite motility within the skin of mice after mosquito challenge (34). Moreover, i.p. administration to human hepatocyte SCID mice of $2.5 \mathrm{mg}$ of an anti-CSP mAb that cross-reacts with $P$. falciparum and $P$. berghei (Pf 49 1B2.2) reduced the number of infected human hepatocytes after i.v. injection of 180,000 P. falciparum sporozoites, but sterile protection was not achieved (35). Here, we found that human anti-CSP mAbs derived from an RTS,S vaccinee were able to prevent infection of human liver uPA-SCID mice by $P$. falciparum when injected prior to parasite challenge, regardless of infection route (i.v. or mosquito bite). The short contact time of antibodies with parasites after nonnatural i.v. injection of sporozoites can conceal the protective effect of antibodies with lower binding affinity that may prove effective when the parasites are delivered via mosquito bite.

The anti-CSP concentrations measured immediately before parasite challenge and induced by administration of $400 \mu \mathrm{g}$ of Mal1C, Mal2A, and Mal3B (Table 1) were in the same range as those previously measured in RTS,S vaccine trials using the same quantification method (25). The lower vaccine efficacy 
Table 1

Prevention of infection by administration of different RTS,S vaccine-induced mAbs

\begin{tabular}{|c|c|c|c|c|c|c|c|}
\hline Anti-CSP mAb (dose) & Mouse ID & $\begin{array}{l}\text { Human albumin } \\
\qquad(\mathrm{mg} / \mathrm{ml})\end{array}$ & $\begin{array}{l}\text { Liver repopulation } \\
\qquad(\%)\end{array}$ & $\begin{array}{l}\text { Weight } \\
\text { (g) }\end{array}$ & $\begin{array}{l}\text { Preinfection titer } \\
(\mathrm{EU} / \mathrm{ml})^{\mathrm{A}}\end{array}$ & $\begin{array}{l}\text { Liver parasite burden } \\
\quad\left(\mathrm{Pf} / 10^{6} \mathrm{HuHEP}\right)\end{array}$ & $\begin{array}{l}\text { Positive } \\
\text { samples }\end{array}$ \\
\hline \multicolumn{8}{|l|}{ i.v. challenge ${ }^{B}$} \\
\hline PBS & B647L & 2.4 & 31.1 & 12.1 & $<50$ & 422.8 & 2 of 12 \\
\hline PBS & K1458RL & 3.5 & 33.2 & 9.7 & $<50$ & 251.6 & 4 of 12 \\
\hline PBS & B627 & 6.8 & 33.5 & 11.8 & $<50$ & $11,991.8$ & 12 of 12 \\
\hline PBS & $\mathrm{B} 625 \mathrm{R}$ & 3.1 & 34.7 & 18.9 & $<50$ & 3,261 & 12 of 12 \\
\hline PBS & B647RL & 6 & 37.9 & 11.7 & $<50$ & $3,233.2$ & 12 of 12 \\
\hline PBS & B673L & 3.9 & 38.7 & 12.1 & $<50$ & 26.9 & 2 of 12 \\
\hline PBS & $\mathrm{B} 644 \mathrm{~L}$ & 6.9 & 59.7 & 10.4 & $<50$ & $2,505.3$ & 12 of 12 \\
\hline Mal1C (2 mg) & B698 & 6.6 & 51.3 & 14.4 & $1,522.9$ & 0 & 0 of 12 \\
\hline Mal1C (2 mg) & B701L & 3.7 & 40.1 & 9.6 & $3,053.1$ & 0 & 0 of 12 \\
\hline Mal1C (2 mg) & $\mathrm{B} 627 \mathrm{R}$ & 4.6 & 32.6 & 12.3 & $3,333.9$ & 0 & 0 of 12 \\
\hline Mal1C (2 mg) & K1447L & 2.5 & 27.7 & 11.2 & $3,513.8$ & 0 & 0 of 12 \\
\hline Mal1C (2 mg) & K1443 & 3.1 & 34.9 & 11.1 & $3,816.0$ & 0 & 0 of 12 \\
\hline Mal1C (2 mg) & K1447 & 4.9 & 47.6 & 12.2 & $4,553.6$ & 0 & 0 of 12 \\
\hline \multicolumn{8}{|c|}{ Mosquito blood meal challenge 1} \\
\hline PBS & K1468L & 4.9 & 37 & 16.5 & $<5$ & 890.7 & 6 of 12 \\
\hline PBS & K1478 & 2.8 & 38.4 & 14.2 & $<5$ & 664.6 & 4 of 12 \\
\hline PBS & K1484L & 5.2 & 55.9 & 15.1 & $<5$ & $1,906.4$ & 10 of 12 \\
\hline PBS & K1482L & 3.8 & 33.1 & 9.5 & $<50$ & $3,637.6$ & 12 of 12 \\
\hline PBS & K1482 & 2.9 & 36.2 & 10.1 & $<50$ & $2,909.4$ & 12 of 12 \\
\hline PBS & K1468L & 9.4 & 52.5 & 13.8 & $<50$ & $1,966.1$ & 11 of 12 \\
\hline Mal1C $(20 \mu \mathrm{g})$ & K1477RL & 7.2 & 61.2 & 11 & 13.1 & 543.5 & 8 of 12 \\
\hline Mal1C $(20 \mu \mathrm{g})$ & B722 & 3.2 & 36.3 & 13.6 & 23.7 & 1,353.2 & 3 of 12 \\
\hline Mal1C $(20 \mu \mathrm{g})$ & B673 & 3.7 & 38.3 & 13.4 & 31.5 & 150.3 & 3 of 12 \\
\hline Mal1C $(200 \mu \mathrm{g})$ & B685 & 3.9 & 42.3 & 16.3 & 139.7 & 0 & 0 of 12 \\
\hline Mal1C $(200 \mu \mathrm{g})$ & K1479L & 3.3 & 30.9 & 15.3 & 230.0 & 29.4 & 2 of 12 \\
\hline Mal1C $(200 \mu \mathrm{g})$ & K1464L & 6.6 & 33.1 & 13.5 & 273.1 & 0 & 0 of 12 \\
\hline Mal1C (2 mg) & B664RL & 6.4 & 51.3 & 14.9 & $2,311.5$ & 0 & 0 of 12 \\
\hline Mal1C (2 mg) & $\mathrm{B} 710 \mathrm{R}$ & 3 & 43.5 & 14.5 & $2,813.6$ & 0 & 0 of 12 \\
\hline Mal1C (2 mg) & B674LL & 3.3 & 36.6 & 11.1 & $3,017.2$ & 0 & 0 of 12 \\
\hline Mal1C (2 mg) & B674L & 4.5 & 33.9 & 11.5 & $4,030.2$ & 0 & 0 of 12 \\
\hline Mal1C (2 mg) & K1475 & 9.2 & 62.9 & 7 & $5,929.1$ & 0 & 0 of 12 \\
\hline \multicolumn{8}{|c|}{ Mosquito blood meal challenge 2} \\
\hline PBS & K1622RL & 1.9 & 29.9 & 11.3 & $<5$ & 623 & 7 of 12 \\
\hline Anti-HBsAg $(400 \mu \mathrm{g})$ & K1651L & 7 & 42.7 & 12.1 & $<5^{C}$ & $4,938.6$ & 11 of 12 \\
\hline Mal1C $(400 \mu \mathrm{g})$ & K1670 & 2.8 & 34.4 & 14.5 & 518.1 & 0 & 0 of 12 \\
\hline Mal1C (400 $\mu \mathrm{g})$ & B894R & 2.1 & 34.5 & 10.9 & 734.3 & 0 & 0 of 12 \\
\hline Mal1C $(400 \mu \mathrm{g})$ & B880 & 4.4 & 43.3 & 8.5 & 784.0 & 0 & 0 of 12 \\
\hline Mal2A $(400 \mu \mathrm{g})$ & K1617L & 5.9 & 47.2 & 12.6 & 400.2 & 0 & 0 of 12 \\
\hline Mal2A $(400 \mu \mathrm{g})$ & B894 & 2.4 & 35.7 & 11.1 & $1,099.9$ & 0 & 0 of 12 \\
\hline Mal2A $(400 \mu \mathrm{g})$ & B880R & 4.2 & 44.2 & 9.2 & 859.1 & 0 & 0 of 12 \\
\hline Mal3B $(400 \mu \mathrm{g})$ & K1617RL & 4.8 & 36.4 & 13.1 & 567.0 & 0 & 0 of 12 \\
\hline Mal3B $(400 \mu \mathrm{g})$ & B864L & 2.8 & 29.2 & 11.8 & 926.0 & 0 & 0 of 12 \\
\hline Mal3B $(400 \mu \mathrm{g})$ & K1552 & 2.1 & 38.7 & 10.2 & $1,247.3$ & 0 & 0 of 12 \\
\hline
\end{tabular}

Pf/106 HuHEP, P. falciparum per $10^{6}$ human hepatocytes. ADepending on dilution, detection limit was 5 or 50 EU/ml. ${ }^{B} 150,000$ sporozoites. CTiter was $1.65 \times 10^{6} \mathrm{mIU} / \mathrm{ml}$

observed in field trials may be due to a progressive decline in antibody titer during the follow-up period. Indeed, after 12 months, a $95 \%$ reduction of anti-CSP titer was observed, and antibody concentrations may drop below the level required to convey sterilizing immunity (36). Our results demonstrated that preventing natural $P$. falciparum infection of humanized mice could be achieved by passive transfer of mAbs induced by
RTS,S vaccination of a malaria-naive volunteer. The sterilizing immunity transferred to the humanized mice provides a proof of principle that anti-CSP antibodies induced by RTS,S are able to prevent $P$. falciparum infection of the liver. These findings further suggest that the protective efficacy of the RTS,S vaccine can possibly be improved by increasing the magnitude and persistence of the CSP-specific antibody response. 


\section{Methods}

Further information can be found in Supplemental Methods and Supplemental Table 1, available online with this article; doi:10.1172/JCI70349DS1.

Generation of humanized mice. Humanized uPA-SCID mice were generated as described previously (5).

Generation of human anti-CSP $m A$ Abs. The cell donor of the PBMCs used to generate the human anti-CSP $\mathrm{mAb}$ was selected from a clinical trial (MAL-080) evaluating the RTS,S vaccine at the Center for Vaccinology, Ghent University and Ghent University Hospital. Human B lymphocytes were immortalized as described previously (37). The mAb concentration was determined by measuring UV absorbance at $280 \mathrm{~nm}(1 \mathrm{mg} / \mathrm{ml}=1.4$ absorbance units) and by anti-CSP ELISA (25).

$m A b$ sequencing. Ig variable genes from 3 anti-CSP-producing hybridomas were sequenced at the Centre for Medical Genetics of Ghent University, and obtained sequences were analyzed using IMGT/V-Quest (http://www.imgt.org/) to assign the variable gene family (33).

Anti-CSP ELISA. Antibodies specific for the CSP tandem repeat epitope were assessed by a validated, standard ELISA (25). At days 0 and 5, mice were bled, and plasma was stored at $-80^{\circ} \mathrm{C}$ until analysis.

In vitro assays of anti-CSP antibody. Gliding assays and inhibition of sporozoite invasion assays were performed as described previously (38).

In vivo parasite challenge and prophylactic treatment experiments. 1 day prior to parasite challenge, chimeric uPA-SCID mice were injected i.p. with PBS, anti-HBsAg $\mathrm{mAb}$, or $\mathrm{mAbs}$ specifically targeting the P. falciparum CSP. The following day, all animals were challenged with sporozoites, either via bites by infected mosquitos or via injection of parasites into the retro-orbital venous sinus. Anopheles stephensi mosquitoes were reared at Radboud University Medical Centre and infected according to previously described standard procedures (39).
Isolation and detection of P. falciparum DNA and human hepatocyte DNA by qPCR. 5 days after infection, mice were euthanized by cervical dislocation, and their livers were stored at $4{ }^{\circ} \mathrm{C}$ until analysis. P. falciparum DNA levels were quantified using a highly sensitive qPCR assay (30). To assess the degree of repopulation with human hepatocytes of the chimeric livers, and to normalize the P. falciparum copy numbers, we used qPCR as previously described (31).

Statistics. Data are shown as mean \pm SD. Analysis were performed using GraphPad Prism.

Study approval. All procedures were approved by the Animal Ethics Committee of the Faculty of Medicine and Health Sciences of Ghent University.

\section{Acknowledgments}

We are grateful to Jolanda Klaassen, Jacqueline Kuhnen, Sofie Librecht, Laura Pelser-Posthumus, Astrid Pouwelsen, Marga van de Vegte-Bolmer, Leen Van Kerkhove, Frédéric Clement, Ivo Ploemen, Peter Vander Linden, Stijn Vanhee, Koen Vercauteren, and Lieven Verhoye for technical assistance. L. Foquet is funded by a PhD grant of the Agency for Innovation by Science and Technology (IWT). This study was funded by Ghent University through a concerted action grant (01G01712) and by the Belgian state (IUAP P7/47HEPRO-2). Anti-CSP human mAbs were a gift of GSK Vaccines.

Received for publication April 5, 2013, and accepted in revised form September 26, 2013.

Address correspondence to: Geert Leroux-Roels, Center for Vaccinology, Ghent University and University Hospital, De Pintelaan 185, 9000 Ghent, Belgium. Phone: 3293323422; Fax: 3293326311; E-mail: geert.lerouxroels@ugent.be.
1. Cohen J, Nussenzweig V, Nussenzweig R, Vekemans J, Leach A. From the circumsporozoite protein to the RTS, S/AS candidate vaccine. Hum Vaccin. 2010 . 6(1):90-96

2. Rosenberg R, Wirtz RA, Schneider I, Burge R. An estimation of the number of malaria sporozoites ejected by a feeding mosquito. Trans $R$ Soc Trop Med Hyg. 1990;84(2):209-212.

3. Jin Y, Kebaier C, Vanderberg J. Direct microscopic quantification of dynamics of plasmodium berghei sporozoite transmission from mosquitoes to mice. Infect Immun. 2007;75(11):5532-5539.

4. Hermsen CC, de Vlas SJ, van Gemert GJ, Telgt DS, Verhage DF, Sauerwein RW. Testing vaccines in human experimental malaria: statistical analysis of parasitemia measured by a quantitative real-time polymerase chain reaction. Am J Trop Med Hyg. 2004; 71(2):196-201.

5. Meuleman P, et al. Morphological and biochemical characterization of a human liver in a UPA-SCID mouse chimera. Hepatology. 2005;41(4):847-856.

6 . Sacci JB Jr, et al. Plasmodium falciparum infection and exoerythrocytic development in mice with chimeric human livers. Int J Parasitol. 2006; 36(3):353-360

7. Vaughan AM, et al. Complete Plasmodium falciparum liver-stage development in liver-chimeric mice. J Clin Invest. 2012;122(10):3618-3628.

8. Bissig KD, Le TT, Woods NB, Verma IM. Repopulation of adult and neonatal mice with human hepatocytes: a chimeric animal model. Proc Natl Acad Sci U S A. 2007;104(51):20507-20511.

9. Azuma H, et al. Robust expansion of human hepatocytes in $\mathrm{Fah}^{-/-} / \mathrm{Rag}^{-/-} / \mathrm{Il} 2 \mathrm{rg}^{-/-}$mice. Nat Biotechnol. 2007;25(8):903-910.

10. Mercer DF, et al. Hepatitis C virus replication in mice with chimeric human livers. Nat Med. 2001; 7(8):927-933.
11. Morosan S, et al. Liver-stage development of Plasmodium falciparum, in a humanized mouse model. J Infect Dis. 2006;193(7):996-1004.

12. Sauerwein RW, Roestenberg M, Moorthy VS. Experimental human challenge infections can accelerate clinical malaria vaccine development. Nat Rev Immunol. 2011;11(1):57-64.

13. Riley E, Stewart V. Immune mechanisms in malaria new insights in vaccine development. Nat Med.2013; 19(2):168-178.

14. Vekemans J, Ballou WR. Plasmodium falciparum malaria vaccines in development. Expert Rev Vaccines. 2008;7(2):223-240.

15. Olotu A, et al. Four-year efficacy of RTS,S/AS01E and its interaction with malaria exposure. $N$ EnglJ Med. 2013;368(12):1111-1120.

16. RTS,S Clinical Trials Partnership, et al. A phase 3 trial of RTS,S/AS01 malaria vaccine in African infants. N Engl J Med. 2012;367(24):2284-2295.

17. Bejon P, et al. Effect of the pre-erythrocytic candidate malaria vaccine RTS,S/AS01E on blood stage immunity in young children. J Infect Dis. 2011; 204(1):9-18.

18. Casares S, Brumeanu TD, Richie TL. The RTS,S malaria vaccine. Vaccine. 2010;28(31):4880-4894.

19. Asante KP, et al. Safety and efficacy of the RTS, $\mathrm{S} / \mathrm{AS} 01$ candidate malaria vaccine given with expanded-programme-on-immunisation vaccines: 19 month follow-up of a randomised, open-label, phase 2 trial. Lancet Infect Dis. 2011;(10):741-749.

20. Abdulla $S$, et al. Safety and immunogenicity of RTS,S/AS02D malaria vaccine in infants. $N$ Engl J Med. 2008;359(24):2533-2544.

21. Kester KE, et al. Randomized, double-blind, phase 2a trial of falciparum malaria vaccines RTS,S/ AS01B RTS,S/AS02A in malaria-naive adults: safety, efficacy, immunologic associates of protection. J Infect Dis. 2009;200(3):337-346.
22. Aponte JJ, et al. Safety of the RTS,S/AS02D candidate malaria vaccine in infants living in a highly endemic area of Mozambique: a double blind randomised controlled phase I/IIb trial. Lancet. 2007; 370(9598):1543-1551.

23. Olotu A, et al. Efficacy of RTS,S/AS01E malaria vaccine exploratory analysis on anti-circumsporozoite antibody titres protection in children aged 5-17 months in Kenya Tanzania: a randomised controlled trial. Lancet Infect Dis. 2011; 11(2):102-109.

24. Moorthy VS, Ballou WR. Immunological mechanisms underlying protection mediated by RTS,S: a review of the available data. MalarJ. 2009;8:312

25. Clement F, et al. Validation of an enzyme-linked immunosorbent assay for the quantification of human IgG directed against the repeat region of the circumsporozoite protein of the parasite Plasmodium falciparum. Malar J. 2012;11:384.

26. Stewart MJ, Nawrot RJ, Schulman S, Vanderberg JP. Plasmodium berghei sporozoite invasion is blocked in vitro by sporozoite-immobilizing antibodies. Infect Immun. 1986;51(3):859-864.

27. Amino R. Host cell traversal is important for progression of the malaria parasite through the dermis to the liver. Cell Host Microbe. 2008;3(2):88-96.

28. House BL, Hollingdale MR, Sacci JB Jr, Richie TL. Functional immunoassays using an in-vitro malaria liver-stage infection model: where do we go from here? Trends Parasitol. 2009;25(11):525-533.

29. Amino R, et al. Quantitative imaging of Plasmodium transmission from mosquito to mammal. Nat Med. 2006;12(2):220-224.

30. Hermsen CC, et al. Detection of Plasmodium falciparum malaria parasites in vivo by real-time quantitative PCR. Mol Biochem Parasitol. 2001; 118(2):247-251

31. Alcoser SY, Kimmel DJ, Borgel SD, Carter JP, 


\section{brief report}

Dougherty KM, Hollingshead MG. Real-time PCR-based assay to quantify the relative amount of human and mouse tissue present in tumor xenografts. BMC Biotechnol. 2011;11:124.

32. Bejon P, et al. Efficacy of RTS,S/AS01E vaccine against malaria in children 5 to 17 months of age. NEngl J Med. 2008;359(24):2521-2532.

33. Brochet X, Lefranc MP, Giudicelli V. IMGT/VQUEST: the highly customized and integrated system for IG and TR standardized V-J and V-D-J sequence analysis. Nucleic Acids Res. 2008;36(Web server issue):W503-W508.
34. Vanderberg J, Frevert U. Intravital microscopy demonstrating antibody-mediated immobilisation of Plasmodium berghei sporozoites injected into skin by mosquitoes. Int J Parasitol. 2004; 34(9):991-996.

35. Sacci JB Jr, et al. Mouse model for exoerythrocytic stages of Plasmodium falciparum malaria parasite. Proc Natl Acad Sci U S A. 1992;89(9):3701-3706.

36. Aide $\mathrm{P}$, et al. Safety, immunogenicity and duration of protection of the RTS,S/AS02(D) malaria vaccine: one year follow-up of a randomized controlled phase I/IIb trial. PLoS One. 2010;5(11):e13838.
37. Depraetere S, Verhoye L, Leclercq G, Leroux-Roels G. Human B cell growth and differentiation in the spleen of immunodeficient mice. J Immunol. 2001; 166(5):2929-2936.

38. van Schaijk BC, et al. Gene disruption of Plasmodium falciparum p52 results in attenuation of malaria liver stage development in cultured primary human hepatocytes. PLoS One. 2008;3(10):e3549.

39. Ponnudurai T, Lensen AH, Van Gemert GJ, Bensink MP, Bolmer M, Meuwissen JH. Infectivity of cultured Plasmodium falciparum gametocytes to mosquitoes. Parasitology. 1989;98(pt 2):165-173. 